\title{
ULRICH NIGGEMANN
}

\section{DIE HUGENOTTEN IN BRANDENBURG-BAYREUTH}

\author{
Immigrationspolitik als »kommunikativer Prozeß«
}

\section{Vorüberlegungen}

Das fränkische Markgraftum Brandenburg-Bayreuth gehörte im Hinblick auf die absolute Zahl der hier angesiedelten Refugiés zu den kleineren Aufnahmeländern. Die historische Forschung geht von etwa 800 bis 1000 französischen Kolonisten aus, die sich unter Markgraf Christian Ernst (geb. 1644, Markgraf seit 1655-1712) im Fürstentum dauerhaft niederließen'. In Relation zu einer Bevölkerung von nur etwa 70000 Einwohnern $^{2}$ war dies jedoch eine durchaus beachtliche Zuwanderung, zumal der Schwerpunkt der Niederlassung der Refugiés eindeutig im sogenannten Unterland, den flächenmäßig kleinen Bayreuther Exklaven um Baiersdorf und Erlangen sowie Neustadt an der Aisch, lag. Im Gegensatz zu anderen Aufnahmeterritorien des deutschen Refuge, insbesondere Brandenburg-Preußen, fehlte es dem Markgraftum an einschlägigen Erfahrungen mit Kolonistengruppen. Im Zuge der konfessionellen Verfolgungs- und Vertreibungsmaßnahmen des 17. Jahrhunderts fanden lediglich einige reformierte Flüchtlinge aus der Oberpfalz sowie vereinzelt Lutheraner aus den Ländern der österreichischen Habsburger Zuflucht in Brandenburg-

${ }^{1}$ Vgl. Gertraud LEHMANN, Gründung und Integration der Französischen Kolonie in Erlangen, in: Christoph FRIEDERICH (Hg.), 300 Jahre Hugenottenstadt Erlangen. Vom Nutzen der Toleranz, Ausstellung im Stadtmuseum Erlangen, 1. Juni bis 23. November 1986, Nürnberg 1986, S. 123-127, hier S. 124; Andreas JAKOB, Die Neustadt Erlangen. Planung und Entstehung, Erlangen 1986 (Erlanger Bausteine zur fränkischen Heimatforschung, Sonderband 33), S. 44. Mit 4000 Kolonisten für Brandenburg-Bayreuth und Brandenburg-Ansbach zusammen setzt Barbara DŐLEMEYER sehr hoch an, möglicherweise unter Einbeziehung weiterer Flüchtlingsgruppen wie etwa der Pfălzer; vgl. DIES., Die Aufnahmeprivilegien fur Hugenotten im europăischen Refuge, in: DIES., Heinz MOHNHAUPT (Hg.), Das Privileg im europăischen Vergleich, Bd. 1, Frankfurt a. M. 1997 (Ius Commune. Sonderhefte, 93), S. 303-328, hier S. 327.

${ }^{2}$ Angabe von 70000 Einwohnern bei Michael PETERS, Wege zur Toleranz. Historische Grundlagen zur Ansiedlung von Hugenotten im Fürstentum Brandenburg-Bayreuth, in: FRIEDERICH (Hg.), 300 Jahre Hugenottenstadt (wie Anm. 1), S. 93-99, hier S. 93. Von etwa 60000 Einwohnern um 1500 und etwa 105000 im Jahr 1754 geht JAKOB, Neustadt (wie Anm. 1), S. 13, aus. 
Bayreuth $^{3}$. Erst die Einwanderung der Hugenotten, deren Beginn mit der Ankunft der ersten Flüchtlingsgruppen im Frühjahr 1686 anzusetzen ist ${ }^{4}$, ist somit als größere, staatlich organisierte Kolonisation zu werten. Ein großer Teil der Refugiés wurde in der eigens zu diesem Zweck erbauten Neustadt Erlangen, ab 1701 Christian-Erlangen, angesiedelt. Weniger bekannt ist jedoch, daß sich Flüchtlinge auch an anderen Orten im Fürstentum, darunter die ländliche Gewerbekolonie Wilhelmsdorf sowie einige Ackerbaukolonien, niederließen ${ }^{5}$.

Die Einzelheiten der Hugenottenansiedlung in der Erlanger Kolonie können als relativ gut erforscht gelten, was nicht zuletzt auf die recht gut greifbaren und geschlossen erhaltenen Quellen zurückzuführen ist ${ }^{6}$. Die »Erlanger Akten « wurden aus wirtschaftshistorischer Sicht bereits 1884 von Georg Schanz und aus theologischer bzw. kirchengeschichtlicher Perspektive 1885 von August Ebrard ausgewertet ${ }^{7}$. Damit war eine realienkundliche Grundlage für eine Reihe weiterer Studien zur Erlanger Kolonie gelegt, die freilich kaum über den von Schanz und Ebrard erarbeiteten Kenntnisstand hinausgingen, jedoch in der

${ }^{3}$ Zur Aufnahme von Oberpfälzer Reformierten in Baiersdorf vgl. Georg SCHANZ, Zur Geschichte der Colonisation und Industrie in Franken, Teil 1, Erlangen 1884 (Bayerische Wirtschafts- und Verwaltungsstudien, 1), S. 7 mit Anm. 4. Zu Flüchtlingen aus Österreich vgl. Georg RUSAM, Österreichische Exulanten in Franken und Schwaben, Neustadt a. d. Aisch ${ }^{2} 1989$ (Einzelarbeiten aus der Kirchengeschichte Bayerns, 63), S. 114. Zur »kolonisatorischen Erfahrung « Brandenburg-Preußens vgl. Matthias ASCHE, Neusiedler im verheerten Land. Kriegsfolgenbewältigung, Migrationssteuerung und Konfessionspolitik im Zeichen des Landeswiederaufbaus. Die Mark Brandenburg nach den Kriegen des 17. Jahrhunderts, Münster 2006, S. 426-436. Asche bezeichnet das Potsdamer Edikt zu Recht als »die Summe kolonisatorischer Erfahrungen des Kurfürsten Friedrich Wilhelm«; ibid., S. 424.

"Vgl. August EBRARD, Christian Ernst von Brandenburg-Baireuth. Die Aufnahme reformirter Flüchtlingsgemeinden in ein lutherisches Land 1686-1712, Gütersloh 1885, S. 22-25; Karl HINTERMEIER, Selbstverwaltungsaufgaben und Rechtsstellung der Franzosen im Rahmen der Erlanger Hugenotten-Kolonisation von 1686 bis 1708, in: Erlanger Bausteine zur fränkischen Heimatforschung 34 (1986), S. 37-162, hier S. 46 (ursprünglich 1948 als Dissertationsdruck erschienen); LEHMANN, Gründung (wie Anm. 1), S. 124.

${ }^{5}$ Hierzu sowie zu weiteren Ansiedlungsorten - auch im Oberland - Johannes E. BISCHOFF, "Hugenotten-Orte« in Franken neben Erlangen, in: FrIEDERICH (Hg.), 300 Jahre Hugenottenstadt (wie Anm. 1), S. 53-58; außerdem die einschlägigen Artikel in DERS., Lexikon deutscher Hugenotten-Orte, Bad Karlshafen 1994 (Geschichtsblätter des Deutschen Hugenotten-Vereins e.V., 22). Zu Wilhelmsdorf auch Rudolf ENDRES, Staat und Gesellschaft, Teil 2: 1500-1800, in: Andreas KRAUS (Hg.), Handbuch der bayerischen Geschichte, Bd. 3/1: Geschichte Frankens bis zum Ausgang des 18. Jahrhunderts, München ${ }^{3} 1997$, S. 702-782, hier S. 765f.; ScHANZ, Geschichte (wie Anm. 3), Teil 1, S. 44-46. Zur ländlichen Ansiedlung ibid., S. 16f.; HINTERMEIER, Selbstverwaltungsaufgaben (wie Anm. 4), S. 46f.

${ }^{6}$ Der Großteil der für die Erlanger Kolonie relevanten Akten befindet sich in den sogenannten "Fasciculi Erlanger Actorum«, Staatsarchiv Bamberg (= StABa) Bestand Geheimes Archiv Bayreuth (= GAB) Nr. 5568-5590.

${ }^{7}$ SCHANZ, Geschichte (wie Anm. 3); EBraRD, Christian Ernst (wie Anm. 4). 
Wertung und Interpretation der Befunde neue Akzente setzten ${ }^{8}$. In größerem Umfang neues Quellenmaterial erschloß hingegen die bauhistorische Untersuchung von Andreas Jakob ${ }^{9}$. Insgesamt fehlt es aber noch an einer plausiblen Charakterisierung der Immigrationspolitik des Markgrafen im Kontext seiner Regierung und seiner Herrschaftsausübung.

Eine solche Einordnung erscheint um so vielversprechender, als die Hugenottenansiedlung in Brandenburg-Bayreuth einige Besonderheiten aufweist, die zugleich eine Reihe genereller Probleme der Immigrationspolitik des 17. Jahrhunderts besonders deutlich hervortreten lassen. Insbesondere die Entwicklung der Privilegien, die die rechtliche, wirtschaftliche und kirchliche Stellung der Kolonie regelten, läßt einen Prozeß der Anpassung der markgräflichen Kolonisationspolitik an die Bedürfnisse der Refugiés erkennen, während sich zugleich im Umgang mit der einheimischen Bevölkerung eine Flexibilität der Regierung zeigt, die Zweifel an einer einseitig auf die Person des Markgrafen ausgerichteten Darstellung der Hugenottenansiedlung aufkommen $1 a ̈ ß t^{10}$. In Anlehnung an neuere Überlegungen zur Konzeptionalisierung von Herrschaft in der Frühen Neuzeit, die sich deutlich von einem einseitigen Modell des Befehls und Gehorsams verabschiedet haben ${ }^{11}$, soll im folgenden die

${ }^{8}$ Unter deutlicherer Betonung auch der Belastungen für die einheimische Bevölkerung vgl. Sigmund vON RAUMER, Erlangen unter Christian und Christian Emst, Erlangen 1910; mit dem Versuch einer rechtshistorischen Einordnung vgl. HINTERMEIER, Selbstverwaltungsaufgaben (wie Anm. 4); mit einem Schwerpunkt auf die Integrationsprozesse und die Entwicklung der Gemeinde vgl. Myriam YARDENI, Refuge und Integration. Der Fall Erlangen, in: Rudolf von THADDEN, Michelle MaGdelaINE (Hg.), Die Hugenotten 1685-1985, München 1985, S. 146-159; sowie die Beiträge in FRIEDERICH (Hg.), 300 Jahre Hugenottenstadt (wie Anm. 1).

${ }^{9}$ JAKOB, Neustadt (wie Anm. 1). Dabei sind insbesondere die Bauakten der Erlanger Neustadt zu nennen, StABa (wie Anm. 6), C60 Nr. 12836, 12878, die auch über die Baugeschichte hinaus wertvolle Informationen über die Organisation der Koloniegründung liefern.

${ }^{10}$ Gerade die ältere Forschung sah - ganz im Sinne des Absolutismus-Konzepts - den Markgrafen zu sehr als allein lenkende und leitende Kraft; vgl. etwa die Darstellung bei SCHANZ, Geschichte (wie Anm. 3), Teil 1, S. 5, 24; oder HINTERMEIER, Selbstverwaltungsaufgaben (wie Anm. 4), S. 41 f., 48 und öfter; sowie im Ganzen VON RAUMER, Erlangen (wie Anm. 8); in der Tendenz ähnlich auch Rudolf ENDRES, Markgraf Christian Ernst von BrandenburgBayreuth, der Gründer der Hugenottenstadt Erlangen, in: Erlanger Bausteine zur fränkischen Heimatforschung 34 (1986), S. 17-35; und JAKOB, Neustadt (wie Anm. 1), S. 11-25.

${ }^{11}$ Vgl. den Diskussionsstand zusammenfassend Markus MEUMANN, Ralf PRÖVE, Die Faszination des Staates und die historische Praxis. Zur Beschreibung von Herrschaftsbeziehungen jenseits teleologischer und dualistischer Begriffsbildungen, in: DIES. (Hg.), Herrschaft in der Frühen Neuzeit. Umrisse eines dynamisch-kommunikativen Prozesses, Münster 2004 (Herrschaft und soziale Systeme in der Frühen Neuzeit, 2), S. 11-49. Vgl. außerdem die Beiträge in Ronald G. ASCH, Heinz DUCHHARDT (Hg.), Der Absolutismus - ein Mythos? Strukturwandel monarchischer Herrschaft in West- und Mitteleuropa (ca. 1550-1700), Köln, Weimar, Wien 1996 (Münsterische Historische Forschungen, 9); Ursula LOFFLER, Dörfliche Amtsträger im Staatswerdungsprozeß der Frühen Neuzeit. Die Vermittlung von Herrschaft auf dem Lande im Herzogtum Magdeburg, 17. und 18. Jahrhundert, Münster 2005 (Herrschaft und 
Immigrationspolitik Markgraf Christian Emsts von Brandenburg-Bayreuth - mit gelegentlichen Seitenblicken auf die Verhältnisse in anderen Territorien - als »kommunikativer Prozeß« beschrieben werden. Die vorliegende Studie geht also von der Annahme aus, daß Herrschaft stets in Kommunikation mit den Beherrschten stand und letztere auf diesem Wege an der Herrschaft teilhatten, weil sie eben nicht nur passive Rezipienten von Informationen und Befehlen waren, sondern die Kommunikationsinhalte aktiv interpretierten und entsprechend agierten ${ }^{12}$. In diesem Sinne läßt sich auch die Ansiedlung französischer Refugiés als ein Vorgang beschreiben, der nicht nur durch die Vorgaben der markgräflichen Regierung bestimmt wurde, sondern auch im Konflikt verschiedener Interessengruppen ausgehandelt wurde.

\section{Die Entstehung und Entwicklung der Privilegien}

Das erste Edikt mit Privilegien für die einwandernden Refugiés erließ Markgraf Christian Ernst am 27. November $1685^{13}$. Der Text des Edikts war das Ergebnis der vorausgegangenen Verhandlungen zwischen der markgräflichen Regierung auf der einen und dem Diplomaten Joseph August du Cros auf der

soziale Systeme in der Frühen Neuzeit, 8), S. 20f.; sowie Dagmar FrEIST, Einleitung: Staatsbildung, lokale Herrschaftsprozesse und kultureller Wandel in der Frühen Neuzeit, in: Ronald G. ASCH, Dagmar FreIST (Hg.), Staatsbildung als kultureller Prozeß. Strukturwandel und Legitimation von Herrschaft in der Frühen Neuzeit, Köln, Weimar, Wien 2005, S. 1-47, hier besonders S. 11-15.

12 Vgl. Ralf Próve, Herrschaft als kommunikativer Prozeß: das Beispiel BrandenburgPreußen, in: DERS., Norbert WINNIGE (Hg.), Wissen ist Macht. Herrschaft und Kommunikation in Brandenburg-Preußen 1600-1850, Berlin 2001 (Schriftenreihe des Forschungsinstituts für die Geschichte Preußens e.V., 2), S. 11-21; MEUMANN, Prove, Faszination (wie Anm. 11), S. 43-49; LOFFLER, Amtsträger (wie Anm. 11), S. 185-189; sowie Stefan BRAKENSIEK, Herrschaftsvermittlung im alten Europa. Praktiken lokaler Justiz, Politik und Verwaltung im internationalen Vergleich, in: DERS., Heide WUNDER (Hg.), Ergebene Diener ihrer Herren? Herrschaftsvermittlung im alten Europa, Köln, Weimar, Wien 2005, S. 1-21, hier S. 1-4. Im Hinblick auf die argumentative Vermittlung von Herrschaftshandeln vgl. auch Andreas GESTRICH, Absolutismus und Öffentlichkeit. Politische Kommunikation in Deutschland zu Beginn des 18. Jahrhunderts, Göttingen 1994 (Kritische Studien zur Geschichtswissenschaft, 103), S. 24-26. Außerdem allgemeiner Alf LOODTKE, Einleitung: Herrschaft als soziale Praxis, in: DERS. (Hg.), Herrschaft als soziale Praxis. Historische und sozialanthropologische Studien, Göttingen 1991 (Veröffentlichungen des Max-Planck-Instituts für Geschichte, 91), S. 9-63.

${ }^{13}$ Les droicts et privilèges que Son Altesse Sérénissime Monseigneur le Marggrave de Brandebourg Bareuth, accorde à tous les François \& autres de la Religion Reformée, qui voudront se venir établir dans ses Etats, Bayreuth, 27. November 1685, StABa GAB (wie Anm. 6), Nr. 5568, fol. 70r-73r, abgedruckt bei SCHANZ, Geschichte (wie Anm. 3), Teil 2, S. 6-8. 
anderen Seite. Du Cros trat dabei als Vertreter der Refugiés auf, stand jedoch in der Folgezeit als Kommissar und Agent in markgräflichen Diensten und kümmerte sich etwa um die Anwerbung von Hugenotten in der Schweiz ${ }^{14}$. Ähnlich wie in Kursachsen gab es im Konsistorium und im Geheimen Rat zu Bayreuth beträchtliche - primär konfessionell begründete - Bedenken gegen die Aufnahme der französischen Kalvinisten in das bis dahin orthodoxlutherisch dominierte Land ${ }^{15}$, doch setzten sich hier die Befürworter der Ansiedlung durch, die vorwiegend an die wirtschaftlichen Vorteile für das Land dachten $^{16}$. Du Cros' Privilegienentwurf, den er am 19. November 1685 dem

${ }^{14}$ Du Cros hielt sich im April 1686 zu Werbungszwecken in der Schweiz auf; vgl. SCHANZ, Geschichte (wie Anm. 3), Teil 1, S. 13f.; PETERS, Wege (wie Anm. 2), S. 98f.; und JAKOB, Neustadt (wie Anm. 1), S. 14. Zur Person du Cros' Harry BRESSLAU, Joseph August du Cros. Ein diplomatischer Abenteurer aus dem Zeitalter Ludwigs XIV., Leipzig 1884; und Michael PETERS, Joseph August du Cros als Agent des Markgrafen Christian Emst von Brandenburg-Bayreuth. Ein Beitrag zur Vorgeschichte der Hugenotten-Kolonisation in Franken, in: Erlanger Bausteine zur fränkischen Heimatforschung 34 (1986), S. 163-174. Allgemein zu den Verhandlungen um die Privilegien, die denselben "Vertragselemente geben hätten, Barbara DOLEMEYER, "Tractat« oder "Begnadigung«? Vertragselemente in Exulantenprivilegien, in: Jean François KERVEGAN, Heinz MOHNHAUPT (Hg.), Gesellschaftliche Freiheit und vertragliche Bindung in Rechtsgeschichte und Philosophie. Zweites deutsch-französisches Symposium vom 12. bis 15. März 1997 in der Herzog-AugustBibliothek Wolfenbüttel, Frankfurt a. M. 1999 (Ius Commune. Sonderhefte 120), S. 144 164, hier S. 154-156.

${ }^{15}$ Bereits 1681, als eine erste Anfrage französischer Protestanten in Bayreuth einging, wehrte das Konsistorium eine Aufnahme der Flüchtlinge erfolgreich ab; Gutachten des Konsistoriums an Markgraf Christian Emst, Bayreuth, 25. November 1681, StABa GAB (wie Anm. 6), Nr. 5568, fol. 3r-6v; abgedruckt bei ScHANZ, Geschichte (wie Anm. 3), Teil 2, S. 2f. Vgl. zu dem Vorgang sowie zur Nichtaufnahme der Hugenotten EBRARD, Christian Ernst (wie Anm. 4), S. 14f.; SCHANZ, Geschichte (wie Anm. 3), Teil 1, S. 7; HINTERMEIER, Selbstverwaltungsaufgaben (wie Anm. 4), S. 42; ENDRES, Markgraf Christian Ernst (wie Anm. 10), S. 24f. Erneute Bedenken des Konsistoriums im Gutachten an Markgraf Christian Ernst, Bayreuth, 20. November 1685, StABa GAB (wie Anm. 6), Nr. 5568, fol. 14r-16v, abgedruckt bei SCHANZ, Geschichte (wie Anm. 3), Teil 2, S. 4f. Vgl. zu dem Gutachten ausführlich EBRARD, Christian Ernst (wie Anm. 4), S. 18-21; sowie SCHANZ, Geschichte (wie Anm. 3), Teil 1, S. 8f. Widerstand im Geheimen Rat leistete v.a. Johann Conrad von Scheres, genannt Zieritz: Schreiben an Markgraf Christian Ernst, Bayreuth, 5. April 1686, StABa GAB (wie Anm. 6), Nr. 5568, fol. 164r-165v; abgedruckt bei SCHANZ, Geschichte (wie Anm. 3), Teil 2, S. 9f. Vgl. dazu Curt HÖFNER, Johann Conrad von Scheres genannt Zieritz, in: Jahrbuch der Coburger Landesstiftung 22 (1977), S. 191-262, hier S. 205f. Zu Kursachsen vgl. den Beitrag von Katharina Middell in diesem Band.

${ }^{16}$ So bereits in der Begründung der Aufnahmeabsicht gegenüber dem Konsistorium durch Markgraf Christian Ernst, Bayreuth, 19. November 1685, StABa GAB (wie Anm. 6), Nr. 5568, fol. 12r-13r; abgedruckt bei SCHANZ, Geschichte (wie Anm. 3), Teil 2, S. 3f. Vgl. zu den Intentionen beispielsweise YARDENI, Refuge (wie Anm. 8), S. 147; oder JAKOB, Neustadt (wie Anm. 1), S. 13. 
Markgrafen vorgelegt hatte ${ }^{17}$, wurde in weiten Teilen in den endgültigen Privilegientext übernommen. Die zentralen Bestimmungen des Textes deckten sich weitgehend mit denen anderer Landesherren ${ }^{18}$ : Die Refugiés wurden dem Schutz des Markgrafen unterstellt und den Einheimischen gleichgestellt. Sie erhielten Vorschüsse für den Aufbau von Manufakturen und Handwerksbetrieben sowie Lebensmittel für die Anfangszeit und Baumaterial für den Aufbau oder die Reparatur von Häusern. Hinzu kamen die Freizügigkeit innerhalb des Territoriums sowie Steuer- und Zollbefreiungen für zehn bzw. fünf Jahre. Interessant sind jedoch gerade die Bestimmungen aus dem Entwurf du Cros', die im Edikt des Markgrafen nicht realisiert wurden. Am auffälligsten ist die Differenz im Bereich der Justiz. Du Cros hatte in seinem Entwurf vom 19. November 1685 drei Artikel zur Rechtsprechung konzipiert, nach denen die Refugiés nicht der Jurisdiktion der örtlichen Richter unterworfen sein, sondern eigene Vermittler und Schiedsrichter zur Schlichtung von Streitfällen haben sollten und ansonsten der Rechtsprechung des Markgrafen und des Geheimen Rats unterstanden hätten ${ }^{19}$. Im Privilegienedikt heißt es dann jedoch:

Le point de la justice étant bien reglé dans l'etat de S[on] $A$ [1tesse], ceux de la religion reformée ne feront pas difficulté de remettre la decision de leurs differens, s'ils ne peuvent étre terminés à l'amiable, au jugement des baillifs dans la jurisdiction desquels ils seront établis ${ }^{20}$.

Nur in Ausnahmefällen sollte es ihnen möglich sein, über einen Bevollmächtigten an den Markgrafen zu appellieren ${ }^{21}$. Dagegen war die Religionsausübung bereits in dem Entwurf du Cros' eingeschränkt. Zwar sollten die Reformierten überall im Land das Recht zu privaten Gottesdiensten haben, doch die öffentliche Ausübung ihrer Religion sollte auf einige bestimmte Orte beschränkt bleiben, was so auch im endgültigen Privilegientext realisiert wurde ${ }^{22}$. Hierin un-

${ }^{17}$ Project du Mons. du Croy pro recipiendis ex Francia profugis Reformatis, 0.O. o.D. (Datierung 19. November 1685 laut Präsentatsvermerk), StABa GAB (wie Anm. 6), Nr. 5568, fol. $17 \mathrm{r}-20 \mathrm{r}$.

${ }^{18}$ Allgemeiner Überblick über die Privilegien anderer Landesherren bei DÖLEMEYER, Aufnahmeprivilegien (wie Anm. 1); Martin PREETZ, Die Privilegien für die deutschen Hugenotten, in: Der Deutsche Hugenott 25 (1961), S. 76-85, 107-123, sowie ibid. 26 (1962), S. 722; und ASCHE, Neusiedler (wie Anm. 3), S. 437-459.

${ }^{19}$ que tous les réformés qui se viendront establir dans ses estats ne dépendront en aucune manière des juges ordinaires des lieux, Artikel 13 des Privilegienentwurfs von du Cros, o.O. o.D. (Präsentatsvermerk vom 9. November 1685), StABa GAB (wie Anm. 6), Nr. 5568, fol. 17r-20r, hier fol. 19r, außerdem Artikel 14 und 15, ibid., fol. 19r-19v. Vgl. auch HINTERMEIER, Selbstverwaltungsaufgaben (wie Anm. 4), S. 53.

${ }^{20}$ Artikel 12 des Edikts von Markgraf Christian Ernst, Bayreuth, 27. Dezember 1685, StABa GAB (wie Anm. 6), Nr. 5568, fol. 70r-73r, abgedruckt bei SCHANZ, Geschichte (wie Anm. 3), Teil 2, S. 6-8.

${ }^{21}$ Ibid. Vgl. auch HINTERMEIER, Selbstverwaltungsaufgaben (wie Anm. 4), S. 53, 58.

22 Artikel 2 bis 4 im Entwurf von du Cros, o.O. o.D. (Präsentatsvermerk vom 19. November 1685), StABa GAB (wie Anm. 6), Nr. 5568, fol. 17r-20r, hier fol. 17r-17v. 
terschieden sich die Bestimmungen deutlich von den Privilegien in HessenKassel und Brandenburg-Preußen, wo das Recht zur öffentlichen Religionsausübung grundsätzlich uneingeschränkt galt ${ }^{23}$. Unklar ist, ob diese Restriktion vom Markgrafen selbst ausging oder ob es sich nicht vielmehr um ein $\mathrm{Zu}$ geständnis gegenüber den strengen lutherischen Kräften im Land handelte.

In beiden Bereichen - in der Rechtsprechung wie auch in der Religionsausübung - wurden die Privilegien in den Folgejahren spezifiziert, modifiziert und erweitert. Die Franzosen selbst, insbesondere - bis zu seiner Entlassung im Oktober 1686 - du Cros, bemühten sich auch nach dem Erlaß der Droicts et privilèges weiter um eine eigenständigere französische Rechtsprechung, wie sie in Brandenburg-Preußen im Potsdamer Edikt angelegt war ${ }^{24}$. So schlug du Cros im September 1686 noch einmal die Errichtung einer chambre de justice sowie eines Handelsgerichts in Erlangen vor, was allerdings erneut abgelehnt wurde ${ }^{25}$. Erst in der »Déclaration« vom 15. August 1687, die das Privilegienedikt von 1685 ergänzte und erweiterte, wurde die Einrichtung eines conseil de justice bestehend aus drei deutschen und drei französischen Richtern angeordnet, die in erster Instanz über alle Streitigkeiten zwischen Deutschen und Franzosen sowie der Franzosen untereinander entscheiden sollten ${ }^{26}$. Anscheinend hatte die Entwicklung in anderen Ländern des deutschen Refuge wie

Mit Nennung von Baiersdorf, wo ihnen erlaubt sein sollte, einen Pfarrer und Schulmeister auf eigene Kosten zu halten, Artikel 3 und 4 des Edikts von Markgraf Christian Ernst, Bayreuth, 27. November 1685, ibid., fol. 70r-73r, abgedruckt bei ScHANZ, Geschichte (wie Anm. 3), Teil 2, S. 6-8.

${ }^{23}$ Relevant ist hier v.a. der Artikel 11 des Edikts Kurfuirst Friedrich Wilhelms, Potsdam, 29. Oktober 1685, Geheimes Staatsarchiv Preußischer Kulturbesitz Berlin-Dahlem (= GStA PK) I. HA Rep. 12232 Nr. 1, fol. 4r-9v, ediert bei Ernst MENGIN, Das Recht der französisch-reformierten Kirche in Preußen. Urkundliche Denkschrift, Berlin 1929, S. 186-195. Für Hessen-Kassel Artikel 12 der Concessions et privilèges Landgraf Karls, Kassel, 12. Dezember 1685, Hessisches Staatsarchiv Marburg (= HStAM) Bestand 5 Nr. 9690, fol. 33r-34r; abgedruckt in Sammlung fürstlich Hessischer Landes-Ordnungen, Teil 3, S. 303-305.

${ }^{24}$ Zur Regelung der Jurisdiktion der brandenburgisch-preußischen Kolonien Artikel 10 des Edikts von Kurfürst Friedrich Wilhelm, Potsdam, 29. Oktober 1685, GStA PK I. HA Rep. 12232 Nr. 1 (wie Anm. 23), fol. 4r-9v, ediert bei MENGIN, Recht (wie Anm. 23), S. 186-195. Vgl. dazu Werner GRIESHAMMER, Studien zur Geschichte der Réfugiés in Brandenburg-Preußen bis 1713, Diss. phil., Berlin 1935, S. 62; und ASCHE, Neusiedler (wie Anm. 3), S. $421 f$.

${ }^{25}$ Du Cros an Markgraf Christian Emst, Frauenaurach, 4. September 1686, StABa GAB (wie Anm. 6), Nr. 5568, fol. 332r-337v. Vgl. dazu und zur Ablehnung durch die Regierung HINTERMEIER, Selbstverwaltungsaufgaben (wie Anm. 4), S. 58f.

${ }^{26}$ Déclaration de Son Altesse Sérénissime Monseigneur Christian Ernest, Markgraff de Brandenbourg [...] concernant les choses, qui doivent être observées parmi les Reformés refugiés de France (Artikel 12), Bayreuth, 15. August 1687, StABa GAB (wie Anm. 6), Nr. 5569, fol. 160r-163r; weitere Exemplare: ibid., Nr. 1r, unfol.; und Stadtarchiv Erlangen (= StAE) Urkunden 15. August 1687; abgedruckt in Corpus Constitutionum BrandenburgicoCulmbacensium, Bd. 2, Abt. 2, Bayreuth 1748, S. 627-638, hier S. 632f. 
auch das Drängen der Koloniemitglieder schließlich doch zu einem Einlenken der markgräflichen Regierung geführt - ein Einlenken, das den Franzosen entgegenkam, zugleich aber einen ersten Schritt zu einer Sondergerichtsbarkeit im Land bedeutete. Hintermeier weist allerdings darauf hin, daß es in der Praxis bereits vor dem Erlaß der Deklaration vom Sommer 1687 erste Schritte in diese Richtung gegeben hatte. In Baiersdorf war es nämlich Mitte des Jahres 1686 zu einem Streitfall gekommen, als eine Quartiergeberin verdächtigt wurde, in das Essen zweier Franzosen, die bei ihr einquartiert waren, Fischangeln gelegt zu haben ${ }^{27}$. Nachdem eine erste Untersuchung vor Ort ergebnislos verlaufen war, ordnete Markgraf Christian Ernst eine weitere Ermittlung unter Hinzuziehung von du Cros, der wegen seiner Abwesenheit durch einen anderen Franzosen namens Viset ersetzt wurde, $a^{28}$. Hintermeier sieht bereits in dieser Einbeziehung eines Franzosen in die ordentliche Gerichtsbarkeit »eine erste Reaktion auf die von den Franzosen mehrfach heftig geübte Kritik an dem für sie nachteiligen Art. XII des Privileges vom 7.12.1685 ${ }^{29}$.

Ein großer Teil der in der Deklaration von 1687 niedergelegten Artikel beschäftigt sich mit den Fragen der reformierten Religionsausübung und stellt somit eine Besonderheit unter den Privilegienedikten dar, die meist nur knapp auf die Frage des Gottesdienstes und der Kirchenverfassung eingehen ${ }^{30}$. Eine

${ }^{27}$ Der Vorfall ist dokumentiert durch die Untersuchungsanordnung Markgraf Christian Ernsts an den Amtmann zu Baiersdorf, Schönfeld, Bayreuth, 3. Juli 1686, StABa GAB (wie Anm. 6), Nr. 5568, fol. 242r-243r; durch den Bericht Schönfelds an Markgraf Christian Ernst, Baiersdorf, 19. Juli 1686, ibid., fol. 244r-245r; und durch das Protokoll des Untersuchungsrichters Panzer, Baiersdorf, 16. Juli 1686, ibid., fol. 246r-247r. Vgl. dazu auch SCHANZ, Geschichte (wie Anm. 3), Teil 1, S. 22 mit Anm. 4; EBRARD, Christian Ernst (wie Anm. 4), S. 27; HINTERMEIER, Selbstverwaltungsaufgaben (wie Anm. 4), S. 67; sowie LEHMANN, Gründung (wie Anm. 1), S. 126. Der Frau konnte übrigens keine Schuld nachgewiesen werden.

${ }^{28}$ Markgraf Christian Ernst an Schönfeld, Bayreuth, 3. Juli 1686, StABa GAB (wie Anm. 6), Nr. 5568, fol. 242r-243r; sowie zur Hinzuziehung Visets Schönfeld an Markgraf Christian Ernst, Baiersdorf, 19. Juli 1686, ibid., fol. 244r-245r.

${ }^{29}$ HINTERMEIER, Selbstverwaltungsaufgaben (wie Anm. 4), S. 67. Datierung im Zitat nach dem Gregorianischen Kalender.

${ }^{30}$ So das Potsdamer Edikt des Kurfürsten Friedrich Wilhelm vom 29. Oktober 1685, das nur in Artikel 11 kurz auf die religiösen Rechte der Refugiés eingeht, GStA PK I. HA Rep. 122 32 Nr. 1 (wie Anm. 23), fol. 4r-9v, abgedruckt bei MENGIN, Recht (wie Anm. 23), S. 186195. Ähnlich auch die Concessions et privilèges Landgraf Karls von Hessen-Kassel, wo Artikel 12 die Haltung von Predigern und den Bau von Kirchen regelt, HStAM Best. 5 Nr. 9690 (wie Anm. 23), fol. 33r-34v. Im Privileg Herzog Ernst Augusts von BraunschweigLüneburg-Calenberg für die Hugenottenkolonie in Hameln finden sich allerdings ähnlich ausführliche Bestimmungen wie im Bayreuther Privileg von 1687, da das Privileg von dem aus markgräflichen Diensten entlassenen und in calenbergische Dienste übernommenen Jean Pierre Ponnier weitgehend identisch übernommen wurde; Privileg für Hameln, Niedersächsisches Hauptstaatsarchiv Hannover (= NHStA) Cal. Br. 8 Nr. 575 Bd. I, fol. 63r-71r; abgedruckt bei Thomas KLINGEBIEL, Die Hugenotten in den welfischen Landen: Eine Privilegi- 
Spezifizierung der die Religionsausübung betreffenden Regelungen erschien aus mehreren Gründen erforderlich. Zum einen war im Dezember 1685 noch keine abschließende Entscheidung über den Hauptansiedlungsort der Hugenotten getroffen worden. Erst im Frühjahr 1686 fiel die Entscheidung für den Neubau einer barocken Planstadtanlage südlich der Altstadt Erlangen ${ }^{31}$. Mit diesem Entschluß erst wurde die Erlaubnis der freien und öffentlichen Religionsausübung sowie der Bau einer Kirche in der Erlanger Neustadt notwendi ${ }^{32}$. Außerdem wurde der öffentliche Gottesdienst in Neustadt an der Aisch, Münchaurach, Stadelhof und einem Vorort von Bayreuth, also weiteren Zentren des Refuge im Fürstentum, gestattet ${ }^{33}$. Zum andern hatten Streitigkeiten um die Religionsausübung genauere Bestimmungen notwendig gemacht. So hatte sich im Mai 1686 der Superintendent zu Baiersdorf, Johann Friedrich Arzberger, beim Konsistorium in Bayreuth beschwert, daß die Franzosen sich in Baiersdorf und Erlangen angemaßt hätten, öffentliche Gottesdienste und Abendmahle abzuhalten $^{34}$. Das Konsistorium erkundigte sich daraufhin beim Markgrafen, welche Rechte die Refugiés in dieser Hinsicht hatten, und erinnerte noch einmal an die Bedenken, die es in seinem Gutachten vom November 1685 vorgebracht hatte $^{35}$. Darüber hinaus war in der Deklaration von 1687 bereits die Einbenufung von Synoden vorgesehen ${ }^{36}$. Erst im Zuge einer größeren gemeindeinternen Auseinandersetzung, die zeitweise eine Auflösung der Kolonie befürchten ließ, setzte sich jedoch in Bayreuth die Erkenntnis durch, daß die Einberufung und Durchführung einer Synode - auch unter Hinzuziehung von Gemeinden

ensammlung, Bad Karlshafen 1994 (Geschichtsblätter des Deutschen Hugenotten-Vereins e.V., 23), S. 62-87.

${ }^{31}$ Vgl. JAKOB, Neustadt (wie Anm. 1), S. 20f., dem zufolge die Entscheidung im April 1686 fiel. Für einen späteren Zeitpunkt spricht, daß Markgraf Christian Ernst Mösch erst am 1. Mai 1686 aufforderte, über geeignete Bauplätze Bericht zu erstatten, StABa GAB (wie Anm. 6), Nr. 5568, fol. 180r-180v; dieser schlug daraufhin Erlangen vor, Mösch an Markgraf Christian Ernst, Erlangen, 4. Mai 1686, ibid., C60 Nr. 12878, fol. 1062r-1066r, hier fol. 1062v.

32 Entsprechende Bestimmung in $\S 1$ der Deklaration von Markgraf Christian Ernst, Bayreuth, 15. August 1687, ibid., GAB Nr. 5569, fol. 160r-163v, Abdruck in Corpus Constitutionum Brandenburgico-Culmbacensium, Bd. 2, Abt. 2, S. 628.

${ }^{33} \S \S 2,3$ und 6 der Deklaration von Markgraf Christian Ernst, Bayreuth, 15. August 1687, StABa GAB (wie Anm. 6), Nr. 5569, fol. 160r-163v, Abdruck in Corpus Constitutionum Brandenburgico-Culmbacensium, Bd. 2 Abt. 2, S. 628-630.

${ }^{34}$ Johann Friedrich Arzberger an das Konsistorium Bayreuth, Baiersdorf, 26. Mai 1686, StABa GAB (wie Anm. 6), Nr. 5568, fol. 187r-188v. Vgl. zu der Beschwerde auch EBRARD, Christian Emst (wie Anm. 4), S. 26; SCHANZ, Geschichte (wie Anm. 3), Teil 1, S. 23.

${ }^{35}$ Konsistorium an Markgraf Christian Ernst, Bayreuth, 3. Juni 1686, StABa GAB (wie Anm. 6), Nr. 5568, fol. 184r-185v.

${ }^{36} \S 5$ der Déclaration Markgraf Christian Ernsts, Bayreuth, 15. August 1687, ibid., Nr. 5569 , fol. $160 \mathrm{r}-163 \mathrm{v}$, Abdruck in Corpus Constitutionum Brandenburgico-Culmbacensium, Bd. 2, Abt. 2, S. 627-638, hier S. 629f. 
aus Brandenburg-Ansbach - am besten geeignet sei, die Probleme zu lösen ${ }^{37}$. Die Möglichkeit, entsprechend der französisch-reformierten Kirchendisziplin Synoden zur Regelung von Fragen oberhalb der Gemeindeebene einzuberufen, war keineswegs in allen Ländern des Refuge gegeben. In den wichtigen Aufnahmeterritorien Brandenburg-Preußen und Hessen-Kassel etwa kam es nicht zu einer solchen Ausformung der Kirchenverwaltung ${ }^{38}$.

Die unklare Rechtslage erforderte also eine Präzisierung und Klarstellung, an die im Vorfeld der Einwanderung noch nicht zu denken gewesen war. Die Forderungen und Streitigkeiten der Refugiés selbst wie auch die Beschwerden einheimischer Institutionen gaben den Ausschlag zu einer Überarbeitung der Rahmenbedingungen, die also erst in der Kommunikation zwischen der Regierung und den betroffenen Personengruppen entwickelt werden konnte.

\section{Die Durchsetzung und Organisation der Einwanderung}

Deutlich wird dieser Aspekt auch in den zahlreichen Konflikten, die in der Frühphase der Einwanderung mit der einheimischen Bevölkerung entstanden. Im Gegensatz zu den im Konsistorium und im Geheimen Rat artikulierten Widerständen gegen die Hugenottenansiedlung lassen sich in den Protesten, Klagen und Beschwerden der einheimischen Bevölkerung keine konfessionellen Motive fassen. Konflikte entstanden in der Gründungsphase der Kolonien - nicht nur in Brandenburg-Bayreuth, sondern generell - primär aufgrund der teilweise

${ }^{37}$ Vorschlag, zur Lösung der Probleme ein Kolloquium einzuberufen, aus unbekannter Feder an Markgraf Christian Ernst, Erlangen, 25. Januar 1688, StABa GAB (wie Anm. 6), Nr. 5570, fol. 320r-327v. Vgl. zu dem Text EBRARD, Christian Ernst (wie Anm. 4), S. 71-73. Vgl. zu den Vorgängen insgesamt SCHANZ, Geschichte (wie Anm. 3), Teil 1, S. 33-39; und sehr ausführlich EBRARD, Christian Ernst (wie Anm. 4), S. 41-74.

${ }^{38} \mathrm{Vgl}$. für Brandenburg-Preußen MENGIN, Recht (wie Anm. 23), S. 5f.; Henri Tollin, Geschichte der Französischen Colonie zu Magdeburg, 6 Bde., Halle a. d. Saale 1886-1892, hier Bd. 2, S. 357f., und Bd. 3/1a, S. 262f.; sowie Thomas KLINGEBIEL, Pietismus und Orthodoxie. Die Landeskirche unter den Kurfürsten und Königen Friedrich I. und Friedrich Wilhelm I. (1688 bis 1740), in: Gerd HenNRICH (Hg.), Tausend Jahre Kirche in Berlin-Brandenburg, Berlin 1999, S. 293-324, hier S. 296. Zu Hessen-Kassel Walter MOGK, Kirchengeschichtliche Aspekte zur Situation der französisch-reformierten Gemeinden im Hessen-Kasselschen Refuge, in: Jochen DESEL, Walter MOGK (Hg.), Die Hugenotten und Waldenser in HessenKassel, Kassel 1978 (Monographia Hassiae, 5), S. 395-435, hier S. 408-415. Anders in den welfischen Territorien, wo sich die französischen Gemeinden mit deutsch-reformierten Gemeinden zur Niedersächsischen Konföderation zusammenschlossen; vgl. dazu Friedrich $\mathrm{H}$. BRANDES, Die Conföderation reformirter Kirchen in Niedersachsen, Magdeburg 1896 (Geschichtsblätter des Deutschen Hugenotten-Vereins, 6. Zehnt, 1/2); und Thomas KLINGEBIEL, Weserfranzosen. Studien zur Geschichte der Hugenottengemeinschaft in Hameln 1690 1757, Göttingen 1992 (Studien zur Kirchengeschichte Niedersachsens, 32), S. 107-115. 
außerordentlichen Belastungen, denen die eingesessenen Untertanen ausgesetzt waren ${ }^{39}$. Die Klagen richteten sich somit nicht in erster Linie gegen die Hugenotten selbst ${ }^{40}$, sondern gegen landesherrliche Anordnungen, und zwar besonders in zwei Bereichen: Erstens wurde gegen die vom Markgrafen angeordneten Fuhrdienste protestiert, die in beträchtlichem Umfang für den Aufbau der Erlanger Neustadt von den fronpflichtigen Untertanen geleistet werden mußten ${ }^{41}$, und zweitens gegen die provisorische Unterbringung der Refugiés durch Einquartierungen in die Häuser der Einheimischen ${ }^{42}$. Dieses Vorgehen der Regierung war keine Besonderheit Brandenburg-Bayreuths, vielmehr wurden

39 Vgl. dazu grundlegend Andreas REINKE, Die Kehrseite der Privilegierung: Proteste und Widerstände gegen die hugenottische Niederlassung in den deutschen Territorialstaaten, in: Comparativ. Leipziger Beiträge zur Universalgeschichte und vergleichenden Gesellschaftsforschung 7, 5/6 (1997), S. 39-52, hier besonders S. 42-47; fast identischer Neuabdruck DERS., "Man fügt ihnen unendlich Schmach zu«. Proteste und Widerstände gegen die Hugenotten in den deutschen Staaten, in: Sabine BENEKE, Hans OTTOMEYER (Hg.), Zuwanderungsland Deutschland: Die Hugenotten [Ausstellung, Deutsches Historisches Museum, Berlin, 22. Oktober 2005 bis 12. Februar 2006], Berlin, Wolfratshausen 2005, S. 65-72, hier besonders S. 66-68. Die von Reinke vorgenommene Einteilung der Konflikte in »Konflikte in der Phase der Ankunft« sowie Konflikte im ländlichen und im städtischen Umfeld ist freilich nicht ganz unproblematisch, da hier eine chronologische Kategorie zwei systematischen Kategorien gegenübergestellt wird und sich die Konflikte in der Ankunftsphase sowohl im städtischen wie auch im ländlichen Umfeld abspielen konnten. Klare Abgrenzungen sind hier nicht möglich.

${ }^{40}$ Hier ist allerdings auch auf Ausnahmen hinzuweisen. In einigen Fällen wurden nämlich Beschwerden gegen das Verhalten der Refugiés vorgebracht, die demnach die Häuser, in denen sie untergebracht waren, gefährdeten, Garten- und Feldfrüchte stahlen, unerlaubt Holz fällten und die Fischteiche ausfischten; so etwa die Klagen von Bürgermeister und Rat zu Erlangen an Markgraf Christian Ernst, Erlangen, 28. Juni 1687, StABa GAB (wie Anm. 6), Nr. 5569, fol. 64r-67r. Ähnlich der Bericht des Amtmanns zu Baiersdorf, Schönfeld, an Markgraf Christian Ernst, Baiersdorf, 25. August 1688, ibid., C60 Nr. 12878, fol. 1095r1095v; oder Bürgermeister und Rat zu Erlangen an Markgraf Christian Ernst, Erlangen, 26. Juni 1694, ibid., GAB Nr. 5571, fol. 316r-317v. Vgl. auch mit Zitat eines Schreibens des Kammerrats Andreas Mösch vON RAUMER, Erlangen (wie Anm. 8), S. 140; sowie JAKOB, Neustadt (wie Anm. 1), S. 52.

${ }^{41}$ Nach einer Angabe des Architekten der Neustadt, Moritz Richter, waren für das Jahr 1687 2000 bis 2600 Fuhren für Quadersteine notwendig. Hinzu kamen 510000 Ziegel und 10000 Stämme Bauholz, die herbeigeschafft werden mußten; Überlegungen und Vorschläge zur Aufteilung der Fuhren durch Mösch an Markgraf Christian Emst mit Memorial Moritz Richters, Plassenburg, 4. November 1686, StABa (wie Anm. 6), C60 Nr. 12878, fol. 321r-324r. Vgl. auch JAKOB, Neustadt (wie Anm. 1), S. 45; und HINTERMEIER, Selbstverwaltungsaufgaben (wie Anm. 4), S. 49.

${ }^{42}$ Die überwiegende Mehrheit der Refugiés wurde in Baiersdorf, Erlangen, Bruck, Frauenaurach und Münchaurach untergebracht, teilweise in herrschaftlichen Gebäuden, größtenteils aber in den Häusern der Untertanen; vgl. SCHANZ, Geschichte (wie Anm. 3), Teil 1, S. 15; EBRARD, Christian Emst (wie Anm. 4), S. 25; HINTERMEIER, Selbstverwaltungsaufgaben (wie Anm. 4), S. 45f.; sowie LEHMANN, Gründung (wie Anm. 1), S. 124. Zur Planung auch eine Denkschrift aus unbekannter Feder, o.O. o.D., StABa GAB (wie Anm. 6), Nr. 5568, fol. $148 r-151 r$. 
ähnliche Maßnahmen auch in Hessen-Kassel und Brandenburg-Preußen ergriffen, wenn auch in unterschiedlichem Ausma $^{43}$.

Bemerkenswerterweise lehnten die von den Diensten und Einquartierungen Betroffenen die Maßnahmen kaum jemals prinzipiell ab, sondern wiesen lediglich auf das Ausmaß der Lasten hin, die sie zu tragen hätten. Insbesondere die zusätzlichen Frondienste während der Erntezeit gaben zu Klagen Anlaß. So schrieb der für die Koordination der Bauarbeiten zuständige Kammerrat Andreas Mösch in einem Bericht an den Markgrafen, daß den Bauern die Fuhrdienste während der Erntezeit sauer und schwer würden ${ }^{44}$. Zudem wurde das Zugvieh stark beansprucht, wie etwa die Klage des Emskirchner Posthalters deutlich macht, der sich beschwerte, daß die Dienste die zu seinem Lebensunterhalt nötigen Arbeiten beeinträchtigten ${ }^{45}$. Im Hinblick auf die Einquartierungen betonten die Bürger der Altstadt Erlangen, daß diese ihnen die gröste beschwernüs bereiteten ${ }^{46}$. Vereinzelt scheint es zu größeren Auseinanderset-

${ }^{43}$ In Hessen-Kassel wurden im Zusammenhang mit dem Aufbau der Landkolonien wie auch der Kasseler Oberneustadt Fuhrdienste von den Einheimischen verlangt. Hinzu kamen Hilfen beim Umpflügen der den Hugenotten zugewiesenen Felder; vgl. Franz-Anton KaDELL, Die Hugenotten in Hessen-Kassel, Darmstadt, Marburg 1980 (Quellen und Forschungen zur hessischen Geschichte, 40), S. 100, 110f., 137-139, 183, 662. Aus Brandenburg-Preußen sind solche Baufuhren hingegen weniger bekannt, doch wurden hier keine größeren Stadtbauprojekte eigens für die Refugiés in Angriff genommen. Dagegen wurden Fuhrdienste v.a. im Zuge der Einwanderung angeordnet; vgl. die Anordnung Kurfürst Friedrich Wilhelms an die Regierung des brandenburgischen Fürstentums Halberstadt, Potsdam, 19. Dezember 1685, GStA PK I. HA Rep. 122 6a Nr. 1 Bd. I (wie Anm. 23), fol. 105r-105v; außerdem TOLLIN, Geschichte (wie Anm. 38), Bd. 2, S. 87. Die provisorische Unterbringung erfolgte auch in Hessen-Kassel teilweise durch Einquartierungen bei der einheimischen Bevölkerung in der Umgebung von Kassel; vgl. Rudolf ScHMIDMaNN, Die Kolonien der Réfugiés in HessenKassel und ihre wirtschaftliche Entwicklung im 17. und 18. Jahrhundert, Diss. phil., Marburg 1929, S. 32f.; KADELL, Hugenotten (wie Anm. 43), S. 73.

${ }^{44}$ Mösch an Markgraf Christian Ernst, Erlangen, 14. Juli 1686, StABa GAB (wie Anm. 6), Nr. 5568, fol. 265r. Vgl. auch VON RAUMER, Erlangen (wie Anm. 8), S. 63; und HINTERMEIER, Selbstverwaltungsaufgaben (wie Anm. 4), S. 49. Ähnliche Klagen wegen der Erntezeit sind auch aus Hessen-Kassel und dem Fürstentum Calenberg bekannt; Bericht Rochaus an Landgraf Karl, Kassel, 13. August 1692, HStAM Best. 5 Nr. 9750 (wie Anm. 23), fol. 53r$57 \mathrm{v}$; und Rentmeister Becker an Landgraf Karl, Kassel, 12. Juli 1688, ibid., Nr. 9832, fol. 273r-273v; sowie für Calenberg Bauschreiber Meyer an den Geheimen Rat zu Hannover, Hameln, 11. Mai 1690, NHStA Cal. Br. 8 Nr. 572 Bd. I (wie Anm. 30), fol. 169r-170r.

${ }^{45}$ Posthalter Bauereiß an Markgraf Christian Emst, Emskirchen, 20. März 1687, StABa GAB (wie Anm. 6), Nr. 5569, fol. 11 r-1lv. Vgl. zu der Klage auch von RAUMER, Erlangen (wie Anm. 8), S. 63; und HINTERMEIER, Selbstverwaltungsaufgaben (wie Anm. 4), S. 49 f. Über Schäden am Zugvieh klagte auch Hans Jacob Hassold, Westheim, 8. März 1687, StABa (wie Anm. 6), C60 Nr. 12878, fol. 569r-570r.

${ }^{46}$ Mit der Klage, daß manch ein Bürger bißweilen 6, 8, 10, 20 auch wohl mehr Franzosen im Haus habe, Bürgermeister und Rat zu Erlangen an Markgraf Christian Ernst, 28. Juni 1687, ibid., GAB Nr. 5569, fol. 64r-67r, hier fol. 65r-65v. Etwa einen Monat später beklagten sich die Bürger erneut über die Einquartierungslast, zumal nun auch noch die Bauarbeiter und eine markgräfliche Leibkompanie zu Pferde untergebracht werden mußten; Bürger und Gast- 
zungen gekommen zu sein. Laut du Cros wurden die Franzosen in einigen Fällen von ihren Quartiergebern schlecht behandelt ${ }^{47}$, und auch die Durchsetzung der Fuhrdienste stieß bisweilen auf Widersetzlichkeiten, etwa als die Untertanen in den Ortschaften Burgbernheim und Bergel zu Materialfuhren herangezogen werden sollten ${ }^{48}$. Ebenso widersetzten sich einige Gemeinden in den Ämtern Hoheneck und Windsheim, so daß der Dachsbacher Amts- und Gerichtsschreiber sich über die unter den Bauern eingerissene unordnung und halßstarrigkeit beklagte ${ }^{49}$. Im Allgemeinen versuchten die Bauern jedoch eher, der Herrschaft Kompromisse anzubieten. So baten sie um Aufschub bei den Fuhren, um ihre Arbeit auf den Feldern erledigen zu können ${ }^{50}$, oder erklärten sich bereit, das, was uf dieselben, nach proportion anderer und größerer Ämter, kommen würde, zu erledigen ${ }^{51}$. Demnach ging es ihnen also darum, im Vergleich zu anderen Ämtern nicht über die Maßen belastet zu werden und eine möglichst gerechte Verteilung der Lasten zu erreichen. In anderen Fällen wurde versucht, eine angemessene Entschädigung zu erhalten. So argumentierten die Bauern in den Ämtern Hoheneck, Windsheim und Altheim, daß ihnen statt des versprochenen Fuhrlohns nur weitere Fuhrdienste zugeteilt worden seien, wodurch sie sich höchst gravirt befinden, weiln gleichwohln die steüern zimblich gro $\beta$ seien. Sollten sie keine Entlohnung erhalten, so seien sie nicht in der Lage, ihre Abgaben zu bezahlen ${ }^{52}$. In ganz ähnlicher Weise versuchten auch

wirte zu Erlangen an Markgraf Christian Ernst, Erlangen, 18. Juli 1687, ibid., fol. 126r$126 \mathrm{v}$.

${ }^{47}$ Du Cros an Markgraf Christian Emst, Baiersdorf, 6. Juni 1686, ibid., Nr. 5570, fol. 51r58v, hier fol. 51v. Vgl. zu diesem Schreiben PETERS, Du Cros (wie Anm. 14), S. 170. Möglicherweise ist auch eine nächtliche handgreifliche Auseinandersetzung im Sommer $1687 \mathrm{im}$ Zusammenhang mit den Einquartierungen zu sehen; vgl. den Bericht von Mösch, o.O. o.D., StABa GAB (wie Anm. 6), Nr. 5569, fol. 68r-73r. Außerdem dazu REINKE, Kehrseite (wie Anm. 39), S. 44; HINTERMEIER, Selbstverwaltungsaufgaben (wie Anm. 4), S. 47f.; und EBRARD, Christian Ernst (wie Anm. 4), S. 27.

${ }^{48}$ Mösch an Markgraf Christian Ernst, Erlangen, 14. Juli 1686, StABa GAB (wie Anm. 6), Nr. 5568, fol. 262r-266r, hier fol. 262r-262v. Vgl. auch SCHANZ, Geschichte (wie Anm. 3), Teil 1, S. 19; und HINTERMEIER, Selbstverwaltungsaufgaben (wie Anm. 4), S. 50.

${ }^{49}$ Zur Widersetzlichkeit der Ämter Hoheneck und Windsheim Bericht des Verwalters von Münchaurach, Adam Krausnach, an Markgraf Christian Ernst, Münchaurach, 7. Juli 1688, StABa (wie Anm. 6), C60 Nr. 12878, fol. 1110r-1110v. Klage des Dachsbacher Amts- und Gerichtsschreibers an Jean Pierre Ponnier, Dachsbach, 19. April 1687, ibid., GAB Nr. 5569, fol. 77r-78r.

${ }^{50}$ Bericht Möschs an Markgraf Christian Ernst, Erlangen, 14. Juli 1686, ibid., Nr. 5568, fol. 262r-266r, hier fol. 262r.

51 Martin Schüffner an Markgraf Christian Ernst, Münchaurach, 5. April 1694, ibid., C60 Nr. 12830, unfol. Ganz ähnlich Johann Wolff Schmidt, Johann Friedrich Köhler, Caspar Ludwig Arzberger, Johann Adam Beck, Johann Achatius Bischoff und Martin Schüffner (wahrscheinlich alles örtliche Beamte) an Markgraf Christian Emst, Emskirchen, 27. April 1694, ibid.

52 So der Bericht des Kastners Johann Daniel Fleischer an Markgraf Christian Ernst, Ipsheim, 17. November 1687 , ibid., Nr. 12878 , fol. $608 \mathrm{r}-608 \mathrm{v}$. 
die von den Einquartierungen betroffenen Bürger der Erlanger Altstadt eine Erleichterung ihrer Lage durch eine gerechtere Verteilung der Lasten zu erreichen. $\mathrm{Da}$ in Erlangen nicht alle Refugiés untergebracht werden könnten, baten sie, einige Flüchtlinge in andere Orte $\mathrm{zu}$ verlegen, wo bis dato noch kein mann logiret worden sei, während dießes kleine städtlein den schwall allein zu tragen habe ${ }^{53}$. Außerdem - so eine weitere Petition - sollten die ebenfalls in der Stadt einquartierten Soldaten in einen Nachbarort umziehen, um Erlangen zu entlasten $^{54}$. Mit dieser Haltung signalisierten die Bürger zweifellos Kompromißbereitschaft und versuchten einen praktikablen Lösungsweg aufzuzeigen, der innerhalb der Grenzen des Erträglichen und Akzeptablen lag ${ }^{55}$.

Zugleich wiesen die Bürger von Erlangen wie auch die Bauern im Umland auf die Basis ihrer wirtschaftlichen Existenz hin. Die Bauern betonten, daß sie während der Erntezeit ihre Feldarbeit zu erledigen hätten, und die Bürger und Gastwirte machten deutlich, daß durch die Überbelegung der Stadt auswärtige Geschäftsreisende keine Bleibe in Erlangen finden könnten, wodurch für unßere nahrung und wirthschafft ein großer mercklicher schade und abgang entstehe ${ }^{56}$. Dies habe, wie die Bauern in den Ämtern Hoheneck, Windsheim und Altheim deutlich machten, auch Folgen für die dem Markgrafen zustehenden Abgaben $^{57}$. Vergleichbare Argumente finden sich auch gegen die Abgabe von Land an die Hugenottenkolonien, etwa als im Zusammenhang mit der Vergabe von Ackerland an einige Refugiés in Wilhelmsdorf eine Müllerin auf bisher als Weide genutztes Land verzichten sollte ${ }^{58}$. Die markgräflichen Untertanen ap-

${ }^{53}$ Bürgermeister und Rat zu Erlangen an Markgraf Christian Emst, Erlangen, 28. Juni 1687, ibid., GAB Nr. 5569, fol. 64r-67r, hier fol. 65v.

${ }^{54}$ Bürger und Gastwirte zu Erlangen an Markgraf Christian Ernst, Erlangen, 18. Juli 1687, ibid., fol. 126r-126v.

${ }^{s s}$ Ein vergleichbares Verhalten läßt sich auch in anderen Territorien nachweisen, so etwa als die Regierung des kurbrandenburgischen Herzogtums Magdeburg die Regierung in Halberstadt bat, nicht alle Flüchtlinge uff Wantzleben, sondern auch einige auf Egeln, Drey und Haymerßleben zu schicken, um eine einseitige Belastung der Amtsuntertanen in Wanzleben zu verhindern, Halle, 7. Januar 1686, Landeshauptarchiv Sachsen-Anhalt in Magdeburg Rep. A 13 Nr. 465, fol. 53r-53v. Vgl. auch Tollin, Geschichte (wie Anm. 38), Bd. 2, S. 11, 110. Auch der Vorschlag, die Fuhren als allgemeine Kriegsfuhren zu deklarieren, entsprang diesem Bestreben, Regienung Halberstadt an Kurfürst Friedrich Wilhelm, Halberstadt, 12. Dezember 1685, GStA PK I. HA Rep. 122 6a Nr. 1 Bd. I (wie Anm. 23), fol. 110r-113r; unkorrekte Darstellung bei TolLIN, Geschichte (wie Anm. 38), Bd. 3/2, S. IV.

${ }^{56}$ Bürger von Erlangen an Markgraf Christian Ernst, Erlangen, 18. Juli 1687, StABa GAB Nr. 5569, fol. 65v.

${ }^{57}$ Bericht des Kastners Johann Daniel Fleischer an Markgraf Christian Ernst, Ipsheim, 17. November 1687, StABa (wie Anm. 6), C60 Nr. 12878, fol. 608r-608v.

${ }^{58}$ Bericht über die Vernehmung der Streitparteien durch Pertsch an Landeshauptmann Pückler, Emskirchen, 23. Juli 1690, ibid., C62 Nr. 2867, unfol. 
pellierten also an ein grundsätzliches gemeinsames Interesse mit ihrem Landesherm an einer Erhaltung ihrer wirtschaftlichen Basis ${ }^{59}$.

Die markgräfliche Regierung hingegen hielt im Grundsatz an ihrem Vorgehen fest, denn die Ansiedlung der Hugenotten hatte für sie Priorität. Zudem sollten die Refugiés so zügig wie möglich von staatlichen Hilfen unabhängig sein, und für den Aufbau der Neustadt Erlangen mußte ein kostengünstiger Weg eingeschlagen werden, zumal die Hugenotten selbst im Gegensatz zu den hochgesteckten Erwartungen wenig Geld ins Land brachten und sich ohne Materiallieferungen nicht zum Häuserbau bereit fanden ${ }^{60}$. Die ehrgeizigen Kolonisationspläne der Regierung wurden daher zu einem Teil auf die Amtsuntertanen abgewälzt, offenbar auch weil es der Regierung vielfach an organisatorischer Weitsicht, solider finanzieller Planung sowie insbesondere am Geld fehlte ${ }^{61}$. Markgraf Christian Ernst drängte deshalb auf eine Beschleunigung der Bauarbeiten und eine zügige Durchführung noch ausstehender Fuhren ${ }^{62}$.

Hinsichtlich der Verteilung der Lasten zeigte die Regierung jedoch Entgegenkommen gegenüber den Klagen aus der Bevölkerung. So schlug Andreas Mösch den Bauern vor, die Fronarbeiten in einem Turnus von zwei Tagen abwechselnd zu verrichten, damit jedem einzelnen Zeit blieb, sich um die eigene Feldarbeit zu kümmern. Außerdem verhandelte er mit den Ämtern über eine gerechte Verteilung der Fuhren ${ }^{63}$. Darüber hinaus wurden Materialfuhren zur Entlastung der Untertanen teilweise mit Hilfe fürstlicher Fuhrwerke durchgeführt, und zur Bezahlung der von den Bauern geleisteten Fuhren wurden im Jahr 1688 feste Sätze fixiert ${ }^{64}$. Auch kam die Regierung der Bitte der Erlanger

${ }^{59} \mathrm{Vgl}$. zu diesem grundsätzlichen Konsens in der ländlichen Wirtschaft LÖFFLER, Amtsträger (wie Anm. 11), S. 133.

${ }^{60}$ Bericht Möschs an Markgraf Christian Ernst, Frauenaurach, 9. Juli 1686, StABa GAB (wie Anm. 6), Nr. 5568, fol. 252r-256r, hier fol. 252r. Vgl. auch SCHANZ, Geschichte (wie Anm. 3), Teil 1, S. 19; und JAKOB, Neustadt (wie Anm. 1), S. 46.

${ }^{61}$ In diesem Sinne etwa JAKOB, Neustadt (wie Anm. 1), S. 53. Zur katastrophalen Finanzlage des Markgraftums vgl. Friedrich SCHUH, Der Markgraf Christian Ernst und die landständische Verfassung des Fürstentums Bayreuth, Borna, Leipzig 1929, S. 53-59; sowie ENDRES, Markgraf Christian Emst (wie Anm. 10), S. 24-26; und DERS., Staat (wie Anm. 5), S. 762-764.

${ }_{62}$ Markgraf Christian Ernst an Mösch, Bayreuth, 4. Juli 1686, StABa GAB (wie Anm. 6), Nr. 5568, fol. 248r; Markgraf Christian Ernst an die Hauptmannschaft Neustadt a. d. Aisch, Erlangen, 22. September 1687, ibid., C60 Nr. 12878, fol. 615r-615v. Außerdem Markgräfin Sophie Louise an den Kastenamtmann zu Baiersdorf, Bayreuth, 31. Oktober 1687, ibid., Rentamt Erlangen Nr. 254a, unfol.

${ }^{63}$ So der Bericht von Mösch an Markgraf Christian Ernst, Erlangen, 14. Juli 1686, ibid., GAB Nr. 5568, fol. 262r-266r, hier fol. 262r. Emskirchen wurde wegen der diesem Amt aufgetragenen Ziegelfuhren von den Holzfuhren ausgenommen, welche dann nach schwierigen Verhandlungen anderen Ämtern zugeteilt wurden; Bericht von Pückler und Mösch an Markgraf Christian Ernst, o.O., o.D., ibid., C60 Nr. 12878, fol. 572r-572v.

${ }^{64}$ Bericht Möschs über die Nutzung von 16 herrschaftlichen Gespannen für die Steinfuhren und Bitte um Aufstockung auf 30 Fuhrwerke, Erlangen, 12. Februar 1687, ibid., fol. 333r334v; sowie Abrechnung der Fuhrkosten, ibid., fol. 331r-332r. Vgl. auch HINTERMEIER, 
Bürger um Verlegung der neben den Refugiés in die Stadt einquartierten Soldaten nach, um so eine Entlastung der Betroffenen zu erreichen ${ }^{65}$. Wegen der Einquartierung der Refugiés in den umliegenden Ortschaften sollte sich du Cros mit den dortigen Einwohnern auf dem Verhandlungsweg einigen ${ }^{66}$. Auch bezüglich der Klage der Müllerin bei Wilhelmsdorf sollten die Beamten zusehen, ob sie nicht mit einem anderen Stück Weideland entschädigt werden könne ${ }^{67}$.

Schon um den sozialen Frieden zu erhalten, wurde ein übereifriges Vorgehen markgräflicher Beamter von seiten der Regierung nach Möglichkeit unterbunden. Wegen seines harten Vorgehens gegen die Widersetzlichkeiten der fronpflichtigen Bauern wurde etwa Ponnier gerügt: Es hätte ihm oblegen, $z u$ sehen, wie mit guter manier und ohne weitleüfftigkeit oder adhibirenden zwang die unterthanen zu denen von ihnen verlangten fuhren zubringen seien $^{68}$. Und ob der Bitte des Windsheimer Kastners um den Einsatz von Musketieren zur Durchführung von Exekutionsmaßnahmen gegen die Orte Burgbernheim, Bergel und Ottenhofen ${ }^{69}$ entsprochen wurde, ist nicht bekannt, aber eher zweifelhaft.

Statt dessen wurde versucht, den Untertanen argumentativ die Intention der Hugenottenansiedlung zu verdeutlichen und um ihr Verständnis und ihre Akzeptanz der Immigrationspolitik zu werben. Am deutlichsten wird dies an der am 6. Dezember 1686 publizierten Erklärung des Markgrafen zum Kolonisationsprojekt, die sich wohl zunächst an die Amtshauptmänner als Multiplikatoren richtete ${ }^{70}$. Darin mahnte der Landesherr zu einem friedlichen Umgang mit den Einwanderern und begründete deren Aufnahme in das Fürstentum mit der gebotenen christliche[n] Liebes-Bezeugung sowie der Hoffnung auf wirtschaftlichen Aufschwung. Die Erklärung, die auch auf die konfessionellen Bedenken seitens der Räte einging, zeigt, daß die Konflikte von der Regierung in Bayreuth durchaus ernstgenommen wurden und daß man sich bewußt war,

Selbstverwaltungsaufgaben (wie Anm. 4), S. 50. Zur Fixierung der Bezahlung vgl. JAKOB, Neustadt (wie Anm. 1), S. 45. Außerdem Bericht Pücklers und Möschs an Markgraf Christian Ernst, o.O. o.D., StABa (wie Anm. 6), C60 Nr. 12878, fol. 572r-572v.

${ }_{65}$ Reskript Markgraf Christian Ernsts an den Capitain der Kompanie, Frauenaurach, 18. Juli 1687, ibid., GAB Nr. 5569, fol. 128r-129r.

${ }^{66}$ Resolution auf den Vorschlag du Cros', die Refugiés gegen Bezahlung in den Dörfern unterzubringen, o.O. o.D. (wohl Juni 1686), ibid., Nr. 5570, fol. 48r-48v.

67 Markgraf Christian Ernst an den Kastner zu Neustadt a. d. Aisch, Bayreuth, 28. August 1690, ibid., C62 Nr. 2867, unfol.

${ }^{68}$ Markgraf Christian Ernst an Ponnier, Bayreuth, 26. April 1687, ibid., GAB Nr. 5569, fol. $79 \mathrm{r}-80 \mathrm{v}$, hier fol. $79 \mathrm{v}$.

${ }^{69}$ Johann Daniel Fleischer an Ponnier, Ipsheim, 6. November 1687, ibid., C60 Nr. 12878, fol. 590r. Offenbar drängte auch Ponnier selbst auf militärische Exekutionsmaßnahmen; vgl. JAKOB, Neustadt (wie Anm. 1), S. 53.

${ }^{70}$ Gedruckter Erlaß, Bayreuth, 6. Dezember 1686, StABa GAB (wie Anm. 6), Nr. 5568, fol. 566r-567v. Weitere Exemplare: ibid., Nr. 1r, unfol.; und StAE (wie Anm. 26), Urkunden 6. Dezember 1686, Abdruck bei ScHANZ, Geschichte (wie Anm. 3), Teil 2, S. 23-25. 
daß ein gewisses Maß an Akzeptanz seitens der Bevölkerung wie auch der lokalen Beamten nötig war, um die Ansiedlung zu verwirklichen.

\section{Einige Schlußfolgerungen}

Die hier skizzierten Beobachtungen aus der Frühphase der Ansiedlung von hugenottischen Refugiés haben gezeigt, daß die Organisation und Durchführung der Immigrationspolitik im kommunikativen Austausch und in der Auseinandersetzung mit den betroffenen Interessengruppen stattfand. Insbesondere die Hugenotten selbst hatten in der Phase der Ausgestaltung ihrer rechtlichen Stellung einen beträchtlichen Einfluß auf die landesherrliche Konzeption. Sie konnten in beachtlichem Maße ihre Vorstellungen mit einbringen und durch die Drohung, das Land wieder zu verlassen oder von vorneherein besseren Angeboten zu folgen, maßgebliche Korrekturen der markgräflichen Entscheidungen erwirken. Doch auch die einheimische Bevölkerung, die durch die Einwanderung und mehr noch durch die Art und Weise, wie dieselbe durchgeführt wurde, in hohem Maße belastet wurde, konnte durch die Artikulation ihrer Interessen in Protest und Widerstand, aber auch in schriftlich eingereichten Petitionen, Klagen und Beschwerden Einfluß auf den Verlauf der Hugenottenansiedlung nehmen. Zwar hatte das Kolonieprojekt für den Landesherren hohe Priorität, so daß keine grundsätzliche Neuausrichtung der Politik stattfand, doch konnten die Einheimischen durchaus Einfluß auf die Umstände nehmen und Belastungen mildern. Tatsächlich zeigt sich in ihrem Agieren, da $B$ sie die Hugenottenansiedlung nicht prinzipiell ablehnten, sondern eher punktuell gegen schwerere Beeinträchtigungen, insbesondere in wirtschaftlicher Hinsicht, protestierten. Gerade diese Haltung ermöglichte es ihnen, Kompromißbereitschaft zu signalisieren und die Kommunikation mit der Herrschaft aufrechtzuerhalten, um so ihre Interessen weiterhin zu artikulieren.

Die Hugenottenansiedlung in Brandenburg-Bayreuth war also kein einsam durch den absolutistischen Fürsten und wenige Berater gelenkter Vorgang, sondern ein Prozeß des Ausprobierens, des Austestens von Grenzen und des Aushandelns gegenläufiger Interessen, mithin also ein »kommunikativer Prozeß« - wie jede Herrschaftsausübung im konkreten Vollzug. Ganz ähnliche Beobachtungen lassen sich auch in anderen deutschen Aufnahmeterritorien machen, wie sich an den hier nur gelegentlich eingestreuten Hinweisen bereits ablesen läßt. Diese Erkenntnis läßt grundlegende Bedenken aufkommen gegen eine Interpretation, wie etwa Andreas Reinke sie hinsichtlich der Konflikte im Refuge huguenot formuliert hat: Reinke zufolge war die Hugenottenansiedlung und -privilegierung seitens der Landesherren auch ein Versuch, ständische 
Strukturen aufzubrechen und bäuerliche wie auch städtische Rechte zu mindern, etwa indem den Bauern zusätzliche Dienste aufgezwungen worden seien ${ }^{71}$. Bei der Durchsicht der Quellen erweist sich jedoch, daß die Ziele aller Beteiligten eher begrenzt waren. Die Landesherren hatten ein deutliches Interesse an der (vermuteten) Wirtschaftskraft der Hugenotten, die sie sich so schnell wie möglich durch eine zügige Etablierung und mit möglichst geringen staatlichen Kosten zunutze machen wollten. Auch die Refugiés selbst waren an einem raschen Aufbau der Kolonien und ihrer Wirtschaftsbetriebe interessiert, darüber hinaus aber auch an einer umfassenden rechtlichen, religiösen und wirtschaftlichen Privilegierung, die von den Landesherren teilweise nur widerstrebend und in mehreren Schritten gewährt wurde. Eben weil die Konflikte um die Hugenottenansiedlung nicht Teil einer größeren und längerfristigen Grundsatzauseinandersetzung zwischen dem werdenden Staat und den traditionellen genossenschaftlichen Strukturen war, waren auch die Ziele der einheimischen Bevölkerung eher begrenzt und punktuell auf Entlastung und Vermeidung größerer Nachteile ausgerichtet. Eben deswegen riß die Kommunikation zwischen Untertanen und Landesherren nicht ab.

\section{Résumé français}

Le margraviat franconien de Brandebourg-Bayreuth comptait parmi les plus petits territoires qui accueillaient les réfugiés français. Une grande partie des réfugiés a été installée dans la Neustadt d'Erlangen spécialement construite à cette fin. L'histoire de la colonie d'Erlangen a déjà été relativement bien étudiée, mais il manque encore une caractérisation de la politique d'intégration du margrave Christian Ernest (1661-1712) sur le fond des nouveaux concepts de recherche concernant les pouvoirs seigneuriaux aux Temps modernes.

Cet article montre que l'arrangement des édits et des privilèges de 1685 et de 1687 ainsi que l'organisation et la réalisation de la politique d'immigration se faisaient à travers un échange communicatif et en discussion avec les réfugiés ainsi qu'avec les groupes de population concernés sur place. Les Français obtinrent que l'on tînt compte de façon considérable de leurs avis dans les privilèges, alors que les protestations de la part de la population locale contre des charges trop lourdes, comme les corvées et le cantonnement, conduisaient dans certains cas à des ajustements de la part du gouvernement. Puisque le projet de colonisation était une priorité majeure pour le margrave, une révision essentielle de cette politique était exclue, d'ailleurs la population ne la revendiquait même pas. Les protestations concernaient plutôt des atteintes concrètes et ponctuelles et non la politique d'accueil des huguenots en général.

En conséquence de ces constatations, force est d'émettre des réserves contre une caractérisation de la politique d'immigration comme action »absolutiste" et dirigée contre les structures de la société des Ordres, idée discutable qui, toutefois, est formulée parfois dans la recherche actuelle. Il s'agit au contraire d'un processus pour tâter le terrain, tester les limites et négocier les intérêts opposés et par conséquent d'un "processus communicatif«.

${ }^{11}$ RENKE, Kehrseite (wie Anm. 39), S. 41, 49f., 54f.; und DERS., Schmach (wie Anm. 39), S. 66, 69f., 72. Ähnlich auch Michael MAURER, Mit Ausländern Staat machen. Glaubensflüchtlinge im Absolutismus, in: Essener Unikate 6/7 (1995), S. 75-85, hier besonders S. 75, 84f. 